\title{
A Micro-Method for Biotinidase Estimation Using Dried Blood Spots and Comparison with Plasma Biotinidase
}

\author{
${ }^{1}$ Iyyapu Krishna Mohan, ${ }^{1}$ K.S.S.Sai Baba, ${ }^{2}$ Chintakindi Krishna Prasad, \\ ${ }^{3}$ Tajamul Hussain ${ }^{3},{ }^{4}$ Salman A Alrokayan, ${ }^{5}$ Shaik Mohammad Naushad, \\ ${ }^{5}$ Radha Rama Devi Akella \\ ${ }^{1}$ Department of Biochemistry, Nizam's Institute of Medical Sciences, Hyderabad, India. \\ ${ }^{2}$ Diagnostic Division, Center for DNA Fingerprinting and Diagnostics, Hyderabad, India. \\ ${ }^{3}$ Center of Excellence in Biotechnology Research, King Saud University, Riyadh,Saudi Arabia; \\ ${ }^{4}$ Department of Biochemistry, College of Science, King Saud University, Riyadh,Saudi Arabia; \\ ${ }^{5}$ Sandor Lifesciences Pvt Ltd, Banjara Hills, Road, Hyderabad.
}

\begin{abstract}
Aims: To develop a micro-method for biotinidase estimation using dried blood spots based on the colorimetric assay developed by Heard et al.

Results: A novel micro-method for biotinidase assay from dried blood spots was developed and validated using Heard et al method for plasma $(n=35)$ and Yamaguchi et al method for dried blood spot $(n=22)$.The correlation coefficients obtained by plotting the values obtained by novel method against the values by Heard et al and Yamaguchi et al methods were 0.86 and 0.90 respectively.

Regression analysis between the novel method and Heard et al method resulted in the following equation for interconversion of values: Plasma Biotinidase in $\mathrm{nmol} / \mathrm{min} / \mathrm{ml}=0.8233+\left[18.4755\right.$ (Blood spot OD $_{540}-$ OD $\left.{ }_{655}\right)$ ]. Biotinidase normal range for plasma was $4.76-10.77 \mathrm{nmol} / \mathrm{min} / \mathrm{ml}$ and for blood spot was 4.52-9.14 $\mathrm{nmol} / \mathrm{min} / \mathrm{ml}$. The intra assay CVs were in the range of $2.8 \%$ to $3.2 \%$ for plasma/serum and 1.5 to $9.3 \%$ for dried blood spots. The inter assay CVs were in the range of 3.3 to $4.7 \%$ for plasma/serum and 2.5 to $9.5 \%$ for dried blood spots. Mean activity in 1000 blood spots of newborns was $6.18 \pm 3.04 \mathrm{nmol} / \mathrm{min} / \mathrm{ml}$.

Conclusion: The modified method can be applied both for newborn screening as well as diagnostic test for biotinidase deficiency.
\end{abstract}

Keywords: biotinidase, diagnostic test, dried blood spots, micro-method, newborn screening, regression analysis

\section{Introduction}

Biotinidase is the enzyme responsible for recycling biotin through biotinyl hydrolase and biotinyl transferase activities that carry out recycling of biocytin to biotin and lysine at acidic $\mathrm{pH}$, transfer of biocytin to nucleophilic acceptor proteins (histones) at physiological or alkaline $\mathrm{pH}$ respectively. [1] Biotin is an essential water-soluble vitamin and is the coenzyme for four carboxylases necessary for normal metabolism in humans: pyruvate carboxylase, propionyl-CoA carboxylase, alpha-methylcrotonyl-CoA carboxylase, and acetyl-CoA carboxylase. [2] Infants with biotinidase deficiency appear normal at birth, but develop critical symptoms after the first weeks or months of life. Symptoms include hypotonia, ataxia, seizures, developmental delay, alopecia, seborrheic dermatitis, hearing loss and optic nerve atrophy. [3,4,5] Metabolic acidosis can result in coma and death.

With early diagnosis and treatment, all symptoms can be prevented. [6] The diagnosis is commonly based on colorimetric method developed by Heard et al using a biocytin analogue, Biotin-4-amido benzoic acid, which is hydrolyzed to 4-amino benzoate in the presence of biotinidase. [7] Yamaguchi et al [8] and Gonzalez et al [9] have developed quantitative and qualitative methods for estimation of biotinidase using dried blood spots. Here we describe a micro-method for quantitation of biotinidase using dried blood spots and compare the results obtained with plasma biotinidase values and Yamaguchi et al method.

\section{Materials And Methods}

Specimens

Plasma and dried blood spots of newborns and infants. 


\section{Methods}

\section{A) Plasma Biotinidase}

Substrate solution containing $0.15 \mathrm{mM}$ Biotin-4-amido benzoic acid in $50 \mathrm{mM}$ potassium phosphate buffer ( $\mathrm{pH}$ : 6.5 ), $0.25 \%$ bovine serum albumin (BSA) and $0.1 \mathrm{mM}$ ethylenediamine tetraacetic acid was prepared. To $100 \mu \mathrm{l}$ of plasma/serum, $800 \mu \mathrm{l}$ substrate solution was added and incubated at $37^{\circ} \mathrm{C}$ for $30 \mathrm{~min}$. The reaction was stopped by adding $200 \mu$ of $30 \%$ tricholoro acetic acid (TCA) and standing for $10 \mathrm{~min}$ completed hemoglobin denaturation. For blank, only substrate alone was incubated and reaction was stopped by addition of TCA. Then plasma/serum was added. Both test and blank tubes were mixed by vortexing followed by centrifugation at $2500 \mathrm{RPM} / 5 \mathrm{~min}$ at $4^{\circ} \mathrm{C}$. Eight hundred $\mu \mathrm{l}$ portions of clear supernatant solution were taken in separate test tubes. For the coupling reaction, $100 \mu \mathrm{l}$ of each of the following reagents was added sequentially in 3 min intervals: $0.1 \%$ sodium nitrite, $0.5 \%$ ammonium sulfamate, $0.1 \% \mathrm{~N}$-1-naphthylethylenediamine dihydrochloride. After $10 \mathrm{~min}$, the absorbance was measured at $540 \mathrm{~nm}$. The molar extinction coefficient for para - amido benzoate is 18.89. A factor of 9.51 was obtained to correct for dilution, incubation time and molar extinction coefficient. Multiplying the difference in the optical densities of test and blank with 9.51 gives the concentration of the test in $\mathrm{nmol} / \mathrm{min} / \mathrm{ml}$. All the samples were tested in duplicates and average of both the assays was considered for final data.

\section{B) Blood Spot Biotinidase}

Blood spots were collected on S\&S 903 filter paper and air dried. Using punching machine $3 \mathrm{~mm}$ discs were punched from each spot and collected in a micro-titer well. Positive and negative controls were also punched similarly and collected in respected micro-wells. To each spot $100 \mu$ l substrate solution was added and incubated at $37^{\circ} \mathrm{C}$ for $6 \mathrm{hrs}$. The reaction mixture was made uniform by shaking on a plate shaker. An aliquot of $60 \mu \mathrm{l}$ was taken into a separate micro-well and the reaction was stopped by adding $60 \mu \mathrm{l}$ of $5 \%$ TCA. Hemoglobin denaturation was completed by incubating for $10 \mathrm{~min}$ at $4{ }^{\circ} \mathrm{C}$. These tubes were centrifuged at 3500 $\mathrm{RPM} / 10 \mathrm{~min}$ at $4^{\circ} \mathrm{C}$. $80 \mu \mathrm{l}$ portions of clear supernatant solution were taken in separate test tubes. For the coupling reaction, $25 \mu \mathrm{l}$ of each of the following reagents was added sequentially in 5 min intervals: $0.1 \%$ sodium nitrite, $0.5 \%$ of ammonium sulfamate, $0.1 \% \mathrm{~N}$-1-naphthylethylenediamine dihydrochloride. After 10min, the absorbance was measured at $540 \mathrm{~nm}$ with background wavelength correction at $655 \mathrm{~nm}$. All the samples were tested in duplicates and average of both the assays was considered for final data.

For complete denaturation of hemoglobin in plasma 30\% TCA is commonly used. While standardizing for blood spot we tried different concentrations such as 30\%, 25\%, 20\%, $15 \%, 10 \%, 5 \%$ and $3 \%$. With concentrations from $30 \%$ to $10 \%$ the intensity of purple color was very high and the optical densities were greater than 1.0 and Beer-Lambert's law cannot be applied. False-positivity was more while using 3\% TCA. 5$7 \%$ TCA was found to be appropriate for blood spot analysis.

\section{Results And Discussion}

Establishing The Correlation Between Plasma Biotinidase And Blood Spot Biotinidase

Based on the values obtained from 35 normal subjects both in plasma and blood spot simple regression analysis was done and an equation was deduced to predict the plasma values for specific blood spot values and vice versa. The equation obtained was as follows:

Plasma Biotinidase in $\mathrm{nmol} / \mathrm{min} / \mathrm{ml}=0.8233+\left[18.4755\left(\right.\right.$ Blood spot $\left.\left.\mathrm{OD}_{540}-\mathrm{OD}{ }_{655}\right)\right]$. The correlation coefficient was 0.86. (Fig 1) The normal range obtained for plasma biotinidase assay was 4.76-10.77 $\mathrm{nmol} / \mathrm{min} / \mathrm{ml}$. The mean activity $\pm \mathrm{SD}$ for normal subjects was $6.94 \pm 2.34 \mathrm{nmol} / \mathrm{min} / \mathrm{ml}$. The normal range of $\mathrm{OD}_{540}-\mathrm{OD}_{655}$ corresponding to blood spot biotinidase activity was 0.2-0.45. Application of the above equation for conversion gives the normal range as $4.52-9.14 \mathrm{nmol} / \mathrm{min} / \mathrm{ml}$, which is fairly in agreement with the plasma values. The mean $\mathrm{OD}_{540}-\mathrm{OD}_{655}$ for normal subjects was 0.33 , which on conversion gives a value of 6.92 $\mathrm{nmol} / \mathrm{min} / \mathrm{ml}$.

\section{Reproducibility}

The intra assay CVs were in the range of $2.8 \%$ to $3.2 \%$ for plasma/serum and 1.5 to $9.3 \%$ for dried blood spots. The inter assay CVs were in the range of 3.3 to $4.7 \%$ for plasma/serum and 2.5 to $9.5 \%$ for dried blood spots.

\section{Stability of Biotinidase activity in Blood Spot}

Dried blood spots from all the 35 normal subjects were periodically analyzed for 10 days in three sets: the first set stored at ambient temperature, the second set stored at $4{ }^{\circ} \mathrm{C}$ and the third set stored at $-20^{\circ} \mathrm{C}$. Paired ttest was conducted taking day 1 data as the reference and day 2, day 3 , day 4, day 5, day 6 , day 7 and day 10 data as the second continuous variable. Differences in the mean values were converted into $\%$ activity of 
biotinidase taking day 1 as $100 \%$. Blood spots stored at ambient temperature and at $4^{\circ} \mathrm{C}$ were stable for 5 days and 7 days respectively. Blood spots stored at $-20^{\circ} \mathrm{C}$ were stable even after 10 days. (Fig 2).

\section{Stability of Reagents}

Sodium nitrite solution was observed degrade faster within 48 hours at ambient temperature and was stable for 5 days at $2-8^{\circ} \mathrm{C}$. Freshly prepared solution improved the quality of the results. Ammonium sulfamate solution stored at $2-8^{\circ} \mathrm{C}$ was found to be stable for several months. A visible change in the color were observed in N-1-naphthylethylenediamine dihydrochloride solution after 4 days at ambient temperature and was stable for 10 days if stored at $2-8^{\circ} \mathrm{C}$ in a dark reagent bottle.

\section{Comparison between Yamaguchi et al method and our method}

\section{Establishing correlation coefficient by plotting both the data on $\mathrm{X}$ and $\mathrm{Y}$ axes}

A set of 23 dried blood spots were tested in duplicates using Yamaguchi's method and the current method. Average biotinidase value of duplicates was considered for data analysis. A graph was plotted with data by Yamaguchi's method on X-axis and data by the current method on Y-axis. The correlation coefficient (r) was 0.90 indicating that the values obtained by both the methods are related.

\section{Measuring agreement between both the methods}

Average biotinidase (OD) by both the methods (X) was plotted against the difference in biotinidase (OD) between both the methods (Y). The mean difference in values was 2.96 with a standard deviation of 1.37. The limits of agreement between these two methods were $0.22,5.7$.

\section{Precision of estimated limits of agreement}

The $95 \%$ confidence interval (CI) for the bias was 1.96 to 3.96 . The $95 \%$ CI for the lower limit of agreement was $-1.50,1.95$. The $95 \%$ CI for the upper limit of agreement was $3.97,7.43$.

\section{Measuring agreement using log transformed data}

When log transformed data was plotted on X- and Y-axes (Yamaguchi's Vs. Our method) the correlation coefficient was 0.90 . The mean difference in log values was 0.19 with a SD of 0.12. Antilog of this value was 1.55. Average of log values was plotted against difference in log values. The limits of agreement between both the methods were $-0.05,0.43$. The $95 \%$ CI for the bias was $0.11,0.27$. The $95 \%$ CI for the lower limit of agreement was $-0.18,0.084$. The $95 \%$ CI for the upper limit of agreement was $0.30,0.56$.

The log-transformed data was converted back to normal by taking antilog. The limits of agreement between both the methods were $0.89,2.69$. The $95 \%$ CI for the bias was $1.28,1.85$. The $95 \%$ CI for the lower limit of agreement was $0.65,1.21$. The $95 \%$ CI for the upper limit of agreement was 1.98, 3.63. The limits of agreement were narrow enough to replace the Yamaguchi's method with the method developed by us. The advantages of the new method over Yamayuchi's method are: (a) $3 / 8^{\text {th }}$ fold reduction in the incubation time of the dried blood spots with the substrate solution, (b) $50 \%$ reduction in the volumes of sodium nitrite, ammonium sulfamate and N-1-Naphthylethylenediamine dihydrochloride reagents used for the color development and (c) background wavelength correction. These three advantages confer that the current method is rapid, costeffective and more specific than the Yamaguchi's method.

\section{Application to Newborn Screening}

Using the modified method, 1000 newborns were screened for biotinidase deficiency and the mean biotinidase activity was $6.18 \pm 3.04 \mathrm{nmol} / \mathrm{min} / \mathrm{ml}$. One case with intermediate activity of $2.21 \mathrm{nmol} / \mathrm{min} / \mathrm{ml} \mathrm{was}$ diagnosed and was supplemented with biotin. On first, second and third follow-up the biotinidase activities were 2.30, 4.33 and $4.65 \mathrm{nmol} / \mathrm{min} / \mathrm{ml}$ respectively indicating good prognosis. Analysis of old blood spots showed false positivity in 29 cases, which were re-tested freshly and found to be normal.

In infants, one 3-month old boy with family history of sibling deaths due to metabolic acidosis and seizures was referred to our center. He was asymptomatic with biotinidase activity $1.1 \mathrm{nmol} / \mathrm{min} / \mathrm{ml}$. After biotin supplementation his first follow-up showed $2.3-\mathrm{nmol} / \mathrm{min} / \mathrm{ml}$ activity. Eighty external quality control samples from CDC, Atlanta over the last four years were analyzed and the results were $100 \%$ satisfactory.

Neonatal screening for biotinidase was started in Virginia in 1984 and later spread to other countries. $[10,11]$ Expanded newborn screening in India was started in 1999 at our center and we are able to screen 3800 newborns using commercially available colorimetric kit and 1000 newborns using this method. [12]. As shown in a recent study, seizure, alopecia and hearing loss are the predominant phenotypes of biotinidase deficient patients [13]. Hypotonia, ataxia, skin rash and seborrhea are the rare presentations. The incidence of biotinidase deficiency in Uttar Pradesh, India was found to be $0.16 \%$ [14]. 


\section{Conclusion}

This method for blood spot biotinidase is rapid, reliable, sensitive, specific, cost-effective and highly reproducible. This can clearly distinguish between a normal and affected person without any ambiguity. Precautions in terms of storage, fresh reagents and proper incubation time can give quality results. Both qualitative and quantitative analysis can be done and results of plasma values can be interpolated. Hence this method is proved suitable for newborn screening as well as routine diagnostics.

\section{References}

[1]. Hymes J, Wolf B. Biotinidase and its roles in biotin metabolism. Clin Chim Acta. 1996 Nov 15;255(1):1-11. Review.

[2]. Wolf B, Grier RE, Allen RJ, Goodman SI, Kien CL. Biotinidase deficiency: the enzymatic defect in late-onset multiple carboxylase deficiency. Clin Chim Acta. 1983 Jul 15;131(3):273-81.

[3]. Wolf B, Grier RE, Allen RJ, Goodman SI, Kien CL, Parker WD, Howell DM, Hurst DL. Phenotypic variation in biotinidase deficiency. J Pediatr. 1983 Aug;103(2):233-7.

[4]. Wolf B, Heard GS, Jefferson LG, Proud VK, Nance WE, Weissbecker KA. Clinical findings in four children with biotinidase deficiency detected through a statewide neonatal screening program. N Engl J Med. 1985 Jul 4;313(1):16-9.

[5]. Bressman S, Fahn S, Eisenberg M, Brin M, Maltese W. Biotin-responsive encephalopathy with myoclonus, ataxia, and seizures. Adv Neurol. 1986;43:119-25.

[6]. Mitchell G, Ogier H, Munnich A, Saudubray JM, Shirrer J, Charpentier C, Rocchiccioli F. Neurological deterioration and lactic acidemia in biotinidase deficiency. A treatable condition mimicking Leigh's disease. Neuropediatrics. 1986 Aug;17(3):129-31.

[7]. Heard GS, Secor McVoy JR, Wolf B. A screening method for biotinidase deficiency in newborns. Clin Chem. 1984 Jan;30(1):1257.

[8]. Yamaguchi A, Fukushi M, Arai O, Mizushima Y, Sato Y, Shimizu Y, Tomidokoro K, Takasugi N. A simple method for quantification of biotinidase activity in dried blood spot and its application to screening of biotinidase deficiency. Tohoku $\mathrm{J}$ Exp Med. 1987 Aug;152(4):339-46.

[9]. Gonzalez EC, Marrero N, Frometa A, Herrera D, Castells E, Perez PL. Qualitative colorimetric ultramicroassay for the detection of biotinidase deficiency in newborns. Clin Chim Acta. 2006 Jul 15;369(1):35-9.

[10]. Heard GS, Wolf B, Jefferson LG, Weissbecker KA, Nance WE, McVoy JR, Napolitano A, Mitchell PL, Lambert FW, Linyear AS. Neonatal screening for biotinidase deficiency: results of a 1-year pilot study. J Pediatr. 1986 Jan; 108(1): 40-6.

[11]. Wolf B. Worldwide survey of neonatal screening for biotinidase deficiency.J Inherit Metab Dis. 1991;14(6):923-7.

[12]. Rama Devi AR, Naushad SM. Newborn screening in India.Indian J Pediatr. 2004 Feb;71(2):157-60.

[13]. Singh A, Lomash A, Pandey S, Kapoor S. Clinical, Biochemical and Outcome Profile of Biotinidase Deficient Patients from Tertiary Centre in Northern India. J Clin Diagn Res. 2015 Dec;9(12):SC08-10.

[14]. Gopalakrishnan V, Joshi K, Phadke S, Dabadghao P, Agarwal M, Das V, Jain S, Gambhir S, Gupta B, Pandey A, Kapoor D, Kumar M, Bhatia V. Newborn screening for congenital hypothyroidism, galactosemia and biotinidase deficiency in Uttar Pradesh, India. Indian Pediatr. 2014 Sep;51(9):701-5.

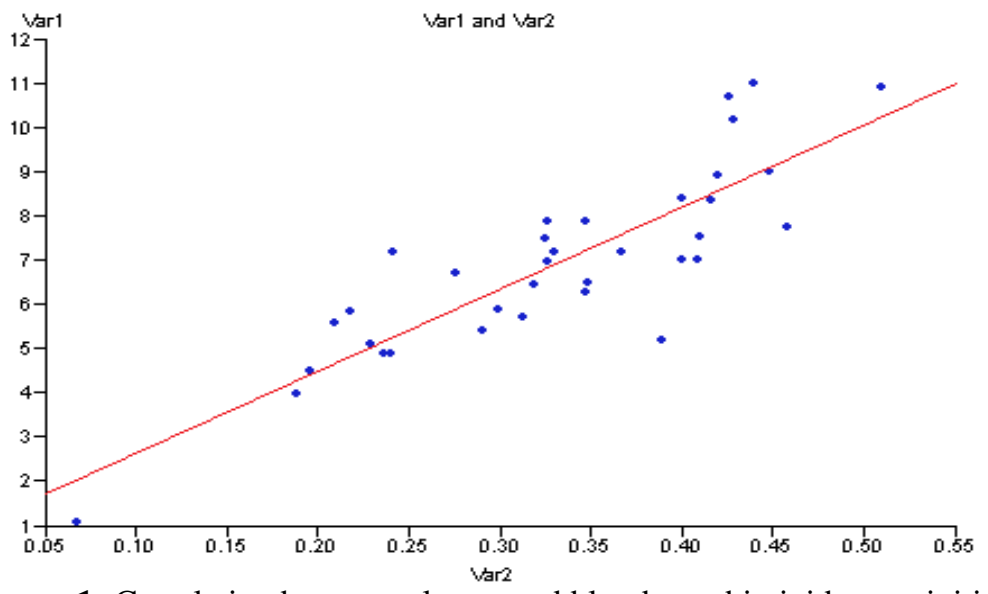

Figure 1: Correlation between plasma and blood spot biotinidase activities

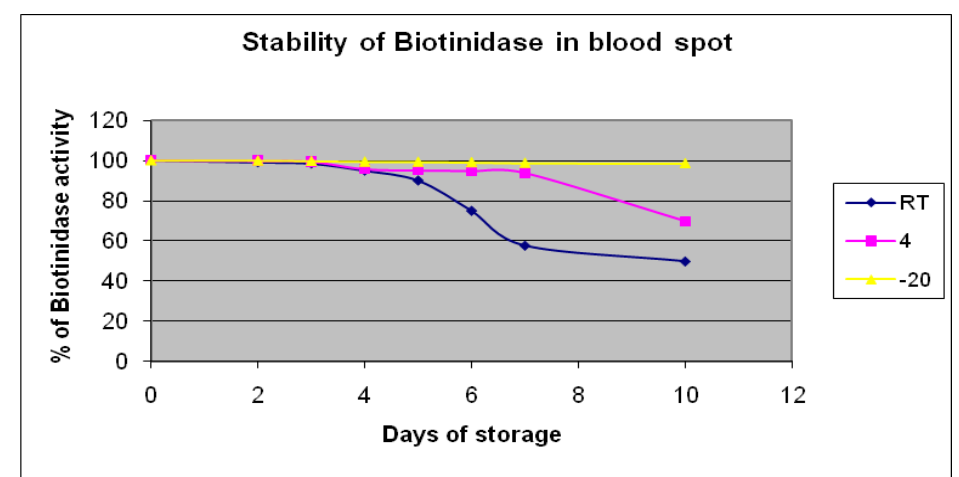

Figure 2. Effect of storage on biotinidase activity in the dried blood spot 